\title{
Erratum to: AtCPK6, a functionally redundant and positive regulator involved in salt/drought stress tolerance in Arabidopsis
}

\author{
Jing Xu • Yong-Sheng Tian • Ri-He Peng • \\ Ai-Sheng Xiong • Bo Zhu • Xiao-Fen Jin • Feng Gao • \\ Xiao-Yan Fu $\cdot$ Xi-Lin Hou $\cdot$ Quan-Hong Yao
}

Published online: 11 July 2010

(C) Springer-Verlag 2010

Erratum to: Planta (2010) 231:1251-1260

DOI 10.1007/s00425-010-1122-0

Unfortunately, the e-mail address and the corresponding author symbol for one of the corresponding authors were not published in the PDF version of the original article.

The complete information is provided below:

X.-L. Hou

State Key Laboratory of Crop Genetics and Germplasm Enhancement, Nanjing Agricultural University, Nanjing 210095, China

e-mail: hxl@njau.edu.cn

The online version of the original article can be found under doi:10.1007/s00425-010-1122-0.

J. Xu · Y.-S. Tian · R.-H. Peng · A.-S. Xiong · B. Zhu •

X.-F. Jin · F. Gao · X.-Y. Fu · Q.-H. Yao (ه)

Agro-Biotechnology Research Institute,

Shanghai Academy of Agricultural Sciences,

2901 Beidi Rd, Shanghai 201106, China

e-mail: yaoquanhong@saas.com.cn;

yaoquanhong_sh@yahoo.com.cn

J. Xu $\cdot$ X.-L. Hou ( $₫)$

State Key Laboratory of Crop Genetics

and Germplasm Enhancement,

Nanjing Agricultural University,

Nanjing 210095, China

e-mail: hxl@njau.edu.cn 